\title{
Isothermal Amplification Technology for Diagnosis of COVID-19: Current Status and Future Prospects
}

\author{
Xuejun $\mathrm{Ma}^{1,2, *}$
}

\begin{abstract}
During the COVID-19 pandemic, polymerase chain reaction (PCR) has become the gold standard for the detection of SARS-CoV-2 RNA worldwide. However, PCR-based nucleic acid detection technology remains relatively timeconsuming, and requires specialized instrumentation and technical personnel; therefore, PCR is difficult to apply at primary-level medical institutions. Antibody-based detection has limitations because of the late appearance of antibodies, thus making early diagnosis difficult, whereas antigen-based detection has insufficient sensitivity, thus resulting in a high false-negative rate. Here, we briefly summarize the development and applications of the nucleic acid isothermal amplification technique (IAT) and describe four major IATs used for the detection of SARS-CoV-2 RNA in mainland China, which have been officially approved by the National Medical Products Administration. In particular, we elaborate on the strengths and weakness of the different IAT in practical settings. We also discuss the outlook for IAT development and propose considerations for the future use of IATs in China.
\end{abstract}

Key words: COVID-19, SARS-CoV-2, isothermal amplification technique
Edited by:

Han Xia, Wuhan Instittue of Virology, Chinese Academy of Science

Reviewed by:

Both reviewers choose to be anonymous

*Corresponding author:

E-mail: maxj@ivdc.chinacdc.cn (XM)

${ }^{1} \mathrm{NHC}$ Key Laboratory of Medical Virology and Viral Diseases, National Institute for Viral Disease Control and Prevention, Chinese Center for Disease Control and Prevention, Beijing, China

${ }^{2}$ Center for Biosafety Mega-Science, Chinese Academy of Sciences, Wuhan, China

Received: November 172021 Revised: January 22022 Accepted: January 72022 Published Online: January 252022
Nucleic acid detection (NAT) technology is widely used for the early detection of infectious-disease pathogens, owing to its advantages of high sensitivity and specificity. Thus, NAT is crucial for the control of infectious disease outbreaks. During the COVID-19 pandemic, polymerase chain reaction (PCR) has become the main technique and gold standard for the detection of SARS-CoV-2 RNA worldwide. However, PCR-based nucleic acid detection technology remains relatively time-consuming, requires specialized instrumentation and technical personnel, is difficult to adopt in rural areas, and is not yet able to meet the needs for rapid testing to facilitate transit through customs ports. Serumspecific $\operatorname{Ig} G$ and $\operatorname{IgM}$ antibody detection, in contrast, has limitations because of the late appearance of antibodies, thus making early diagnosis difficult. Antigen detection has the drawbacks of insufficient sensitivity and a high false-negative rate $[1,2]$. The emerging nucleic acid isothermal amplification technique (IAT) overcomes the shortcomings of PCR technology, through its advantages of simple operation, rapid detection, and accurate results. Therefore, IAT is more suitable for customs ports requiring rapid detection and for primary institutions (such as fever clinics) at the county and rural levels, which may lack technical expertise and have limited human resources. Personnel training for IAT detection of SARS-CoV-2 RNA can be completed within a short time [3-5]. 
Currently, most commonly used IATs include nucleic acid sequence-based amplification (NASBA) [6], loop-mediated amplification (LAMP) [7], recombinase polymerase amplification (RPA) [8], and novel IATs based on Cas enzyme-based IATs, such as specific high sensitivity enzymatic reporter unlocking (SHERLOCK) [9] and DNA endonuclease-targeted CRISPR trans reporter (DETECTR) [10]. The Cas enzyme system, a highly specific nucleic acid sequence identification and reporter method, can improve the accuracy of detection results when combined with the above-mentioned IATs. SHERLOCK and DETECTR have been successfully used for SARS-CoV-2 RNA detection, with a detection sensitivity of ten copies per reaction within $1 \mathrm{~h}$ [11]. However, both methods require the tube to be opened for amplification-product transfer at the end of the reaction, and the tube must be opened again for test strip detection, thereby increasing the risk of product contamination and operational difficulties. To solve this problem, a Cas12bbased assay, SHERLOCK testing in one pot (STOP), has been developed. The Cas12b used in STOP can maintain its activity at the LAMP reaction temperature $\left(65^{\circ} \mathrm{C}\right)$, thus eliminating the need to open the tube after amplification to transfer the product and decreasing the risk of product contamination [12]. Although the Cas system has become a major technical research focus, most CRISPR-Cas-based detection methods are typically combined with a prior amplification reaction to achieve sensitive detection, thus complicating the procedures. Several studies have recently reported the elimination of the pre-amplification process by using tandem CRISPR nucleases [13] or CRISPRCas13a and mobile-phone microscopy [14] for amplification-free detection of SARS-CoV-2. In addition, the Cas detection system has no selective specificity for the cleavage of reporter probes, and the development of single-tube multiplex detection faces technical challenges.

Four main IATs, simultaneous amplification and testing (SAT; Register Certificate No. 20193220245) [15], recombinase-aided amplification (RAA; Register Certificate No. 20213400656) [16,17], cross-priming amplification (CPA; Register Certificate No. 20203400241), and LAMP centrifugal disc-based microfluidic chip (LAMP-chip; Register Certificate No. 20203400178) [18] have been officially approved by the National Medical Products Administration for the detection of SARS-CoV-2 RNA in mainland China. SAT, with a fully automated system for nucleic acid extraction (AutoSAT), can achieve high-throughput detection of 200 samples in $8 \mathrm{~h}$. However, AutoSAT does not overcome the problem of the long reaction time of NASBA. In addition, SAT still requires routine viral nucleic acid extraction of samples in advance and is therefore best suited for high-throughput SARS-CoV-2 RNA detection in laboratory settings in quarantine and inspection departments. Real-time fluorescence RAA can be used to detect nucleic acids in many sample types, such as throat swabs, nasopharyngeal swabs, nasal swabs, and alveolar lavage, with high sensitivity (200 copies/mL) and specificity. The detection of 16 samples per run can be completed within 8-15 minutes after the addition of the extracted nucleic acid. Equipped with automated nucleic acid extraction instruments, the entire process for 96 samples can be completed within $1 \mathrm{~h}$, and the detection results can be automatically interpreted with a portable isothermal amplification fluorescence instrument. With its rapidity, simple operation and flexibility, RAA is best suited for rapid screening at county and township fever clinics and port testing facilities [16]. Real-time fluorescence CPA uses an all-in-one, fully automated system for nucleic acid detection. The CPA amplification products are detected with molecular beacons, and the fluorescence signal is detected by an internal fluorescence detection device. This fully automated detection tube is suitable for on-site screening in early stages of an outbreak. LAMP-chip is used for the detection of six respiratory pathogens, including SARS-CoV-2 [19]. The primers corresponding to the six viruses on the chip are preloaded in different primer preloading units; during the assay, the mixture of extracted nucleic acid and the reagents are added separately and evenly distributed in the reaction unit under the action of centrifugal force. The chip is reacted at $63^{\circ} \mathrm{C}$ for $1.5 \mathrm{~h}$ to complete the amplification of 16 samples, and the amplified products are detected with a detection instrument. LAMP-chip enables the differential detection of multiple respiratory pathogens and is suitable for rapid triage of patients with indications of respiratory diseases at medical institutions. However, this method requires a traditional viral nucleic acid extraction step, which limits the speed of detection and is unsuitable for rapid on-site detection. Previous studies have reported that LAMP is relatively more resistant to inhibitors and can be used for the direct detection of blood samples with simple lysis treatment [20] or of swab samples with a boiling water-bath treatment [21]. The development of direct detection of thermolyzed pharyngeal swab samples will facilitate rapid detection of SARS-CoV-2 in the field.

The IATs described above are based on different principles and product detection methods, and consequently differ in their sensitivity, specificity, required instrumentation, and detection time. The tests must be selected according to different scenarios (Table 1). Although IATs exhibit some advantages over PCR in the detection speed, instrument miniaturization, reagent cost, and visual interpretation of results, field investigation remains difficult to achieve, because sample pre-treatment (nucleic acid extraction) has become a major constraint. Using a simple sample-processing method and pairing it with the proper IAT will be crucial for practical application in the field. Second, achieving multiplex or multi-target IAT detection in a single closed-tube format with high sensitivity and specificity is also an urgent technical obstacle that must be addressed to avoid limiting the scope of IAT applications. Some IAT point-of-care test devices achieve the ultimate goal of providing results for tested samples, solve the difficulties of nucleic acid extraction and multiplex detection, and show good prospects for field application; however, the applications of the current 
TABLE 1 | Comparison of isothermal amplification techniques.

\begin{tabular}{|c|c|c|c|c|c|c|c|c|}
\hline Method & Template & $\begin{array}{l}\text { Amplification } \\
\text { time (min) }\end{array}$ & $\begin{array}{l}\text { Limit of } \\
\text { detection } \\
\text { (copies) }\end{array}$ & $\begin{array}{l}\text { Reaction } \\
\text { temperature }\left({ }^{\circ} \mathrm{C}\right)\end{array}$ & $\begin{array}{l}\text { Number of } \\
\text { primers }\end{array}$ & $\begin{array}{l}\text { One-tube } \\
\text { multiple } \\
\text { detection }\end{array}$ & $\begin{array}{l}\text { Result determination } \\
\text { method }\end{array}$ & $\begin{array}{l}\text { Country of } \\
\text { Inventor }\end{array}$ \\
\hline NASBA & RNA & 60 & 1 & $41(65 / 95)$ & 2 & Difficult & $\begin{array}{l}\text { Fluorescence detection, } \\
\text { test strip }\end{array}$ & Canada \\
\hline SAT & RNA & 90 & 1 & $41(65 / 95)$ & 2 & Difficult & Fluorescence detection & China \\
\hline LAMP & DNA or RNA & 60 & 5 & $60-65$ & $4-6$ & Difficult & $\begin{array}{l}\text { dye, Fluorescence } \\
\text { detection, turbidity }\end{array}$ & Japan \\
\hline LAMP-chip & DNA or RNA & 90 & 15 & 63 & $4-6$ & Possible & $\begin{array}{l}\text { Fluorescence detection, } \\
\text { dye }\end{array}$ & China \\
\hline RPA & DNA or RNA & $5-15$ & 1 & $37-42$ & 2 & Difficult & $\begin{array}{l}\text { Fluorescence detection, } \\
\text { test strip }\end{array}$ & UK \\
\hline RAA & DNA or RNA & $5-15$ & 1 & $37-42$ & 2 & Difficult & $\begin{array}{l}\text { Fluorescence detection, } \\
\text { test strip }\end{array}$ & China \\
\hline CPA & DNA or RNA & 60 & 4 & 63 & $2-4$ & Difficult & $\begin{array}{l}\text { Fluorescence detection, } \\
\text { test strip }\end{array}$ & China \\
\hline SHERLOCK & DNA or RNA & 50 & 10 & $37-42$ & 2 & Difficult & $\begin{array}{l}\text { Fluorescence detection, } \\
\text { test strip }\end{array}$ & USA \\
\hline DETECTR & DNA or RNA & 40 & 10 & $60-65$ & $4-6$ & Difficult & $\begin{array}{l}\text { Fluorescence detection, } \\
\text { test strip }\end{array}$ & USA \\
\hline
\end{tabular}

products are limited by their relatively low throughput, high cost, long assay times, or low sensitivity.

In the future, NAT technology is expected to move toward the development of digitalization, miniaturization, simplification, field use, and automation. IATs still cannot replace PCR in pathogen detection, but they compensate for shortcomings of traditional PCR including equipment dependence and long detection time. Therefore, the IAT development trend is expected to make these assays valuable for rapid detection of infectious pathogens and fully automated detection. Microfluidic chips, a new reaction and detection vehicle, can be combined with various IATs to develop a compact, portable platform for bedside detection or instant detection. Microfluidic chips can also integrate multiple reactions to achieve simultaneous detection of multiple samples or multiple targets. The major challenge faced by this method is avoiding compromising clinical performance in the integration of nucleic acid extraction, amplification, and detection steps in a single chip. Fabrication cost is another major concern, although recently emerging mobile-phone- and paper-based microfluidic devices provide attractive alternatives in resource-poor settings [22,23]. Real-world field applications of IATs also require consideration of miniaturization of the instrument or visual interpretation without instrumentation, affordable assay costs, smart data transfer (e.g., with smartphones), and battery-powered devices.

Because strategies for prevention and control vary among countries, SARS-CoV-2 may coexist with humans or could even become a transmitted respiratory pathogen for long time periods. In the face of this serious situation, external prevention of viral importation and internal prevention of rebound remain the focus of controlling the COVID-19 pandemic in China. With the gradual opening of the country's borders during the post-vaccine period, the pressure for external prevention of importation will continue to increase, and the capacity for rapid nucleic acid testing at customs sites must be improved as soon as possible. Another challenge lies in the prevention of internal rebound, given that China's health-services network has fewer resources in rural areas. In this regard, IAT detection of SARS-CoV-2 RNA has the potential to be promoted and applied at the grassroots and customs levels.

In practical settings, combined detection might be optimal, if feasible. For example, IATs could be used for primary screening, and PCR could then be used for confirmation and review of the results, thus taking full advantage of both technologies to meet the practical needs of rapid screening at ports and rapid triage at grassroots fever clinics. Furthermore, given that the development and industrialization of domestic IATs is growing faster in China than in other countries, we suggest setting up a special project for research and development of new technologies, encouraging exploratory and original research, and focusing on supporting the development and industrialization of new IATs with independent intellectual-property rights.

\section{ACKNOWLEDGEMENTS}

We gratefully acknowledge assistance from the National Health Commission of the People's Republic of China and the Chinese Center for Disease Control and Prevention.

\section{CONFLICTS OF INTEREST}

The authors has no conflicts of interest to declare. 


\section{REFERENCES}

1. Aguilar-Shea AL, Vera-García M, Güerri-Fernández R. Rapid antigen tests for the detection of SARS-CoV-2: a narrative review. Aten Primaria. 2021;53(9):102127.

2. Li D, Li J. Immunologic testing for SARS-CoV-2 infection from the antigen perspective. J Clin Microbiol. 2021;59(5):e02160-20.

3. Zhao Y, Chen F, Li Q, Wang L, Fan C. Isothermal amplification of nucleic acids. Chem Rev. 2015;115(22):12491-12545.

4. Craw $P$, Balachandran W. Isothermal nucleic acid amplification technologies for point-of-care diagnostics: a critical review. Lab Chip. 2012;12(14):2469-2486.

5. Li J, Macdonald J. Advances in isothermal amplification: novel strategies inspired by biological processes. Biosens Bioelectron. 2015;64:196-211.

6. Compton J. Nucleic acid sequence-based amplification. Nature. 1991;350(6313):91-92

7. Notomi T, Okayama H, Masubuchi H, Yonekawa T, Watanabe $\mathrm{K}$, Amino N, et al. Loop-mediated isothermal amplification of DNA. Nucleic Acids Res. 2000;28(12):E63.

8. Piepenburg O, Williams $\mathrm{CH}$, Stemple DL, Armes NA. DNA detection using recombination proteins. PLOS Biol. 2006;4(7):e204.

9. Gootenberg JS, Abudayyeh OO, Lee JW, Essletzbichler P, Dy AJ, Joung J, et al. Nucleic acid detection with CRISPR-Cas13a/C2c2. Science. 2017;356(6336):438-442.

10. Chen JS, Ma E, Harrington LB, Da Costa M, Tian X, Palefsky JM, et al. CRISPR-Cas12a target binding unleashes indiscriminate single-stranded DNase activity. Science. 2018;360(6387):436-439.

11. Broughton JP, Deng $X, Y u$ G, Fasching $C L$, Servellita $V$, Singh J, et al. CRISPR-Cas12-based detection of SARS-CoV-2. Nat Biotechnol. 2020;38(7):870-874.

12. Joung J, Ladha A, Saito M, Segel M, Bruneau R, Huang $M-l$, et al. Point-of-care testing for COVID-19 using SHERLOCK diagnostics. medRxiv [Preprint]. 2020 May 8:2020.05.04.20091231. DOI:10.1101/2020.05.04.20091231.

13. Liu TY, Knott GJ, Smock DCJ, Desmarais JJ, Son S, Bhuiya $A$, et al. Accelerated RNA detection using tandem CRISPR nucleases. Nat Chem Biol. 2021;17(9):982-988.
14. Fozouni P, Son S, Díaz de León Derby M, Knott GJ, Gray CN, D'Ambrosio MV, et al. Amplification-free detection of SARSCoV-2 with CRISPR-Cas13a and mobile phone microscopy. Cell. 2021;184(2):323-333.e9.

15. Li T, Shi T, Sun Y, Zhou K, Huang Z, Wang P, et al. Application of real-time simultaneous amplification and testing method to accurately and rapidly detect extra-pulmonary tuberculosis. BMC Infect Dis. 2020;20(1):303.

16. Wang J, Cai K, He X, Shen X, Wang J, Liu J, et al. Multiple-centre clinical evaluation of an ultrafast singletube assay for SARS-CoV-2 RNA. Clin Microbiol Infect. 2020;26(8):1076-1081.

17. Chen C, Li XN, Li GX, Zhao L, Duan SX, Yan TF, et al. Use of a rapid reverse-transcription recombinase aided amplification assay for respiratory syncytial virus detection. Diagn Microbiol Infect Dis. 2018;90(2):90-95.

18. Yuan X, Lv J, Lin X, Zhang C, Deng J, Wang C, et al. Multiplex detection of six swine viruses on an integrated centrifugal disk using loop-mediated isothermal amplification. J Vet Diagn Invest. 2019;31(3):415-425.

19. Li B, Zou BJ, Ma XP, Wu HP, Zhang YJ, Zhou GH. Research progress in technologies based on isothermal amplification of nucleic acids for detection of SARS-CoV-2. Chinese J Virol. 2021 DOI:10.13242/j.cnki.bingduxuebao.003857.

20. Dixit KK, Verma S, Singh OP, Singh D, Singh AP, Gupta R, et al. Validation of SYBR green I based closed tube loop mediated isothermal amplification (LAMP) assay and simplified direct-blood-lysis (DBL)-LAMP assay for diagnosis of visceral leishmaniasis (VL). PLoS Negl Trop Dis. 2018;12(11): e0006922.

21. Mikita K, Maeda T, Yoshikawa S, Ono T, Miyahira Y, Kawana A. The Direct Boil-LAMP method: a simple and rapid diagnostic method for cutaneous leishmaniasis. Parasitol Int. 2014;63(6):785-789.

22. Ji T, Liu Z, Wang G, Guo X, Akbar Khan S, Lai C, et al. Detection of COVID-19: a review of the current literature and future perspectives. Biosens Bioelectron. 2020;166:112455.

23. Mauk M, Song J, Bau HH, Gross R, Bushman FD, Collman RG, et al. Miniaturized devices for point of care molecular detection of HIV. Lab Chip. 2017;17(3):382-394. 